\title{
Hábitos alimentarios, actividad física y bimomio ansiedadV depresión como factores de riesgo asociados con el desarrollo de obesidad en población militar
}

\author{
Vazquez Guzman MA. ${ }^{1}$
}

Sanid. mil. 2014; 70 (1): 6-12; ISSN: 1887-8571

\begin{abstract}
RESUMEN
Antecedentes y objetivos: El sobrepeso y la obesidad son reconocidos como uno de los retos de salud pública del mundo. El objetivo fue determinar si los hábitos alimentarios, actividad física, y ansiedad y depresión, son factores de riesgo asociados con el desarrollo de obesidad en población militar mexicana. Material y métodos: Se realizó un estudio observacional, analítico, tipo casos y controles, en el que se determinó la prevalencia de sobrepeso-obesidad, definida como sujetos con un IMC>27, y la fuerza de asociación entre dieta hipercalórica, actividad física moderada, ansiedad y depresión con su desarrollo. Se incluyeron 50 casos y 50 controles pareados por jerarquía (1:1). El análisis estadístico se realizó mediante $\mathrm{X}^{2}$ y Odds Ratio (OR) crudas y ajustadas por jerarquía militar. Resultados: De la población de 3.000 militares masculinos en el activo, se obtuvo una muestra de 189, que tuvo una prevalencia de $26.5 \%$. Se observó diferencia entre casos y controles para la edad $(\mathrm{z}=3,69)$, peso $(\mathrm{z}=8,73)$ e IMC $(\mathrm{z}=8,72)$. La actividad física moderada fue un factor protector para el desarrollo de obesidad $(X 2=13,07 ; p=<0,001 ; O R=0,19 ; \mathrm{IC} 95 \%$ de 0,07 a 0,48$)$. La dieta hipercalórica fue un factor de riesgo para el desarrollo de la misma $(X 2=25,49 ; \mathrm{p}=<0,001 ; \mathrm{OR}=9,68 ; \mathrm{IC} 95 \%$ de 3,80 a 24,65). Para la tropa, la depresión fue un factor de riesgo $(X 2=3,95 ; \mathrm{p}=<0,05 ; \mathrm{OR}=2,52 ; \mathrm{IC} 95 \%$ de 1,00 a 6,33). La ansiedad y la asistencia al comedor no fueron factores de riesgo. Los menús del comedor de la brigada excedieron en $905 \mathrm{kcal}$. a las necesidades energéticas. Conclusiones: La depresión y la dieta hipercalórica fueron factores de riesgo para el desarrollo de sobrepeso-obesidad y la actividad física actuó como factor protector. La importancia de este estudio radica en que es el primero con éstas características que se realiza en población militar mexicana, por ello sienta las bases para no seguir dependiendo de la información de otras poblaciones, permitiéndonos hacer intervenciones más objetivas.
\end{abstract}

PALABRAS CLAVE: Obesidad, Casos y controles, Actividad física moderada, Dieta hipercalórica, Depresión.

Eating habits, physical activity and binomio anxiety / depression as risk factors associated with the development of obesity in soldiers. SUMMARY: Background and objectives: Overweight and obesity are recognized as one of the public health challenges of the world. The objective was to determine whether dietary habits, physical activity, and anxiety and depression are risk factors associated with the development of obesity in Mexican's soldiers. Material and methods: We performed an observational, analytical, case-control, in which we determined the prevalence of obesity, defined as subjects with a BMI> 27, and the strength of association between calorie diet, moderate physical activity, anxiety and depression with development. There were 50 cases and 50 matched controls hierarchy (1:1). Statistical analysis was performed using $\mathrm{X}^{2}$ and Odds Ratio (OR) and adjusted raw military ranks. Results: In 3000 the population of the active male soldiers, we obtained a sample of 189 , with a prevalence of $26.5 \%$. Difference was observed between cases and controls for age $(z=3.69)$, weight $(z=8.73)$ and BMI $(z=8.72)$. Moderate physical activity was a protective factor for the development of obesity ( $\mathrm{X}^{2}=13.07, \mathrm{p}=<0.001, \mathrm{OR}=0.19,95 \% \mathrm{CI}$ from 0.07 to 0.48$)$. Hypercaloric diet was a risk factor for the development of the same $\left(\mathrm{X}^{2}=25.49, \mathrm{p}=<0.001, \mathrm{OR}=9.68,95 \% \mathrm{CI}\right.$ from 3.80 to 24.65$)$. For privates, depression was a risk factor $\left(\mathrm{X}^{2}=3.95, \mathrm{p}=<0.05\right.$, $\mathrm{OR}=2.52,95 \% \mathrm{CI}$ from 1.00 to 6.33). Anxiety and dining assistance were not risk factors. Dining menus brigade exceeded $905 \mathrm{kcal}$. energy needs. Conclusions: Depression and hypercaloric diet were risk factors to the development of overweight and obesity and exercise was a protective factor. The importance of this study is that it is the first with these characteristics carried out in Mexican military population, so the basis for no longer rely on information from other populations, allowing us to make more objective interventions.

KEYWORDS: Obesity, Case-control, Moderate physical activity, Calorie diet, depression.

\section{INTRODUCCIÓN}

La obesidad es una enfermedad de etiología multifactorial y curso crónico en la cual se involucran tanto aspectos

${ }^{1}$ Mayor Médico Cirujano. Escuela Militar de Graduados de Sanidad. México.

Dirección para correspondencia: Dr. Miguel Antonio Vazquez Guzman. Escuela Militar de Graduados de Sanidad, Calle Cerrada de palomas, S/N, Colonia Lomas de Sotelo, México, Distrito Federal, C.P. 11200.medivazquez@gmail.com

Recibido: 15 de enero de 2013

Aceptado: 14 de octubre de 2013 genéticos como ambientales y de estilo de vida que conducen a un trastorno metabólico, caracterizada por una acumulación excesiva de la grasa corporal, originada por un balance positivo de energía, el cual ocurre cuando la ingesta de calorías excede al gasto energético, lo cual origina ganancia de peso $^{1-4}$.

La Encuesta Nacional de Salud y Nutrición en México del año 2006 (ENSANUT 2006) registró que al sumar las prevalencias de sobrepeso y obesidad, el 71,9\% de las mujeres mayores de 20 años de edad y el $66,7 \%$ de los hombres presentaban exceso de peso $^{5-9}$. 
Actualmente se consideran factores de riesgo asociados al desarrollo de la enfermedad determinantes genéticos, de estilo de vida y socioeconómicos ${ }^{10-12}$.

La depresión y la ansiedad también se han asociado con el desarrollo de obesidad, según estudios recientes realizados en Estados Unidos de América, en América Latina y en México ${ }^{13-15}$.

En relación al estilo de vida, la falta de actividad física regular también se ha definido como factor importante de riesgo para el desarrollo de las principales enfermedades crónicas no transmisibles, incluida la obesidad ${ }^{16-18}$.

Según la Norma Oficial Mexicana «Para el manejo integral de la Obesidad» (NOM-174-SSA1-1998) se diagnostica Obesidad cuando el IMC es mayor de $27 \mathrm{~kg} / \mathrm{m}^{2}$ en personas de talla normal y mayor de $25 \mathrm{~kg} / \mathrm{m}^{2}$ en personas de talla baja ${ }^{19,20}$.

En México, el coste total del sobrepeso y la obesidad (suma del coste indirecto y directo) ha aumentado desde 35.429 millones de pesos en el año 2000 al estimado de 67.345 millones de pesos en 2008. La proyección para el 2017 asciende a 150.860 millones de pesos 9 .

En el Ejército mexicano, se han realizado varios estudios en relación a esta enfermedad ${ }^{21}$. Un estudio descriptivo realizado por Chávez en 1996, en mujeres cadetes de la Escuela Militar de Enfermeras, observó una prevalencia de $32,34 \%$ de obesidad, y concluyó que los menús del servicio de alimentación estaban mal calculados y proporcionaban una dieta hipercalórica a esta población ${ }^{22}$.

En 1998 un estudio analítico, realizado por López en militares en el activo de la Primera Brigada de Ingenieros, mostró una prevalencia de obesidad mayor que la prevalencia nacional $(24 \%$ contra 21,5\% respectivamente). En adición, este estudio, reportó no haber encontrado asociación de la obesidad y el sedentarismo $\left(\mathrm{X}^{2}=2,38\right)$. Similar al estudio de Chávez, se puso de manifiesto que los menús eran hipercalóricos para esa población ${ }^{23}$. Un estudio tipo casos y controles, realizado en militares atendidos en el Hospital Central Militar de México, por Lizárraga, en el año 2007 , encontró que un IMC superior a $25 \mathrm{~kg} / \mathrm{m}^{2}$ se asoció con el desarrollo de hipercolesterolemia, hiperuricemia e hipertensión arterial sistémica ${ }^{24}$. Por último, un estudio descriptivo, realizado en el año 2007, en pacientes con obesidad de la Unidad de Especialidades Médicas del ejército mexicano, por Reyes, reportó una prevalencia de ansiedad superior a la nacional (47.4 contra $16 \%$ respectivamente) y una prevalencia de depresión similar a la nacional ( $18 \%$ contra $20 \%$ respectivamente $)^{25}$.

\section{MATERIAL Y MÉTODOS}

Se realizó un estudio observacional, analítico, tipo casos y controles, en el que se determinó la prevalencia de obesidad, y la fuerza de asociación entre dieta hipercalórica, actividad física moderada, ansiedad y depresión con el desarrollo de obesidad.

La población de estudio fue de 3.000 militares en servicio activo de la Segunda Brigada de Infantería del Campo Militar 1-A, Ciudad de México, cada participante firmó una carta de consentimiento informado.

La muestra se obtuvo mediante la fórmula $n=p \times q /(E P /$ $\mathrm{Z})^{2}$. Sin embargo, para obtener porcentajes exactos se elevó «n» a más de 100 unidades estadísticas, elegidas mediante muestreo aleatorio simple por medio de la lista nominal de la Brigada, resultando una $n=189$, a la cual se le aplicó un cuestionario diseñado por el investigador, validado y ajustado (Alfa de Cronbach= 0.787 y Coeficiente de correlación intraclase $=78 \%)^{26-31}$.

Se obtuvieron sus datos antropométricos (peso y talla) y se establecieron los grupos, considerando como casos (militares con obesidad) a los encuestados que tuvieron IMC $\geq 27 \mathrm{~kg} / \mathrm{m}^{2}$ ó $\geq 25 \mathrm{~kg} / \mathrm{m}^{2}$ con talla baja (estatura menor de $1.60 \mathrm{~m}$ ) y como controles a los militares en servicio activo sin obesidad (IMC < $27 \mathrm{~kg} / \mathrm{m}^{2} \mathrm{o}<25 \mathrm{~kg} / \mathrm{m}^{2}$ con talla baja) $)^{5,19}$. Se encontraron 50 casos y se compararon con el mismo número de controles, pareados por jerarquía $(1: 1)$.

Para la determinación de niveles de actividad física se consideró el tipo de ejercicio (realizados en 30 minutos y por lo menos 3 veces por semana) y se establecieron 3 categorías: 1) Ligera: caminar. 2) Moderada: carrera, natación, bicicleta a velocidad baja, baile, baloncesto, voley- ball, futbol. 3) Intensa: realización de ejercicio con objetos muy pesados, cavar, realizar aerobic, spining, o bicicleta a velocidad elevada ${ }^{5}$.

Se consideró la dieta como hipercalórica si la cantidad de calorías consumida en promedio durante dos días (Laboral y Domingo) excedió a la cantidad de calorías calculadas por la fórmula de Harris-Benedict para varones ${ }^{23,35,36}$, ajustada según el grado de actividad física: Sedentario (ninguna actividad (1'2), Ligera (1'5), Moderada (1'7) o Intensa (2'2) y sumado al efecto térmico de los alimentos $(7,5 \%$ de la cantidad de calorías promedio de los 2 días $)^{5}$.

Se consideró ansiedad como diagnóstico cuando el sujeto obtuvo puntuación mayor a 7 en la escala de ansiedad y depresión hospitalaria (HAD), utilizando la subescala para ansiedad, y depresión como diagnóstico cuando el sujeto obtuvo puntuación mayor a 7 en la escala de ansiedad y depresión hospitalaria (HAD), utilizando la subescala para depresión ${ }^{32}$.

La asociación entre exposición y enfermedad, se exploró con la prueba Chi-cuadrada $\left(\mathrm{X}^{2}\right)$, y la fuerza de asociación entre la exposición al factor sospechoso y el desarrollo de obesidad, mediante la Odds Ratio (OR), utilizando el paquete estadístico SPSS (Statistical Product and Service Solutions) versión $1733-35$.

Tabla 1. Características de los casos (obesidad) y controles en la $2^{a}$ Brigada de infantería, marzo-abril, 2010.

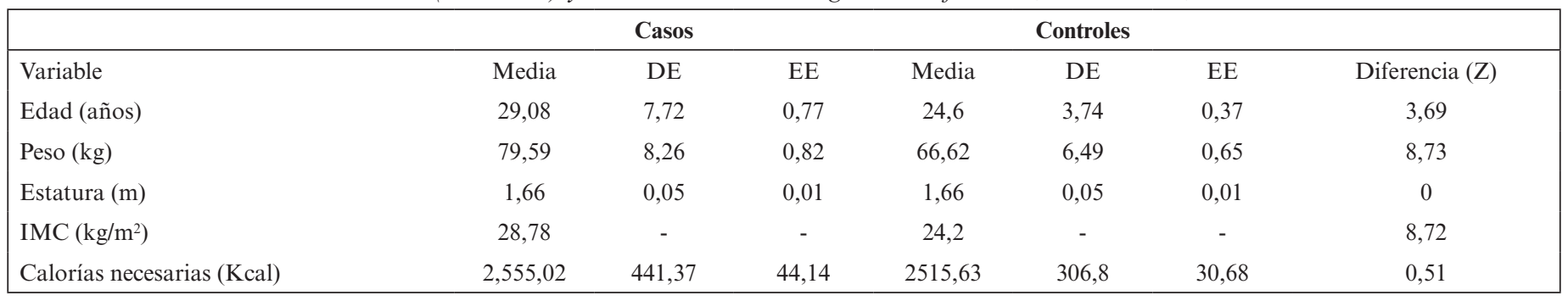


Hábitos alimentarios, actividad física y binomio ansiedad/depresión como factores de riesgo ...

Los menús del comedor se evaluaron mediante el programa informático NUTRIBER con el fin de valorar si eran adecuados para las necesidades energéticas de los militares de esta población ${ }^{36}$.

\section{RESULTADOS}

La población total de la Segunda Brigada de Infantería estuvo formada por 3.000 militares masculinos en el activo; la muestra estudiada de 189 , tuvo una prevalencia de 26 ' 5 por cada 100 personas. Se observó que existió diferencia entre los casos y controles para la media de las variables de edad (29'08 contra $24^{\prime} 6$ respectivamente, $\left.z=3^{\prime} 69\right)$, peso (79'9 contra 66'62 respectivamente, $\left.z=8^{\prime} 73\right)$ e índice de masa corporal (28'7 contra $24^{\prime} 2$ respectivamente, $\left.z=8^{\prime} 72\right)$, no así en las variables de estatura y calorías necesarias (Tabla 1).

Por otro lado, se observó que existió diferencia entre los casos y controles para la prevalencia de las variables de dieta hipercalórica ( $68 \%$ contra $18 \%$ respectivamente, $z=5 \% 85)$ y actividad física moderada ( $50 \%$ contra $84 \%$ respectivamente, $z=3$ ' 87 ), no así para las variables de depresión, ansiedad y asistencia al comedor institucional (Tabla 2).

Tabla 2. Prevalencia de las varables en los casos (obesidad) y controles en la $2^{a}$ Brigada de infantería, marzo-abril, 2010.

\begin{tabular}{|lccc|}
\hline Variable & $\begin{array}{c}\text { Prevalencia } \\
\text { casos (\%) }\end{array}$ & $\begin{array}{c}\text { Prevalencia } \\
\text { controles (\%) }\end{array}$ & Diferencia (z) \\
\hline Depresión & $68 \%$ & $54 \%$ & 1,45 \\
Ansiedad & $36 \%$ & $38 \%$ & 0,20 \\
Dieta hipercalórica & $68 \%$ & $18 \%$ & 5,85 \\
Actividad física moderada & $50 \%$ & $84 \%$ & 3,87 \\
Asistencia al comedor & $64 \%$ & $62 \%$ & 0,20 \\
\hline
\end{tabular}

La realización de actividad física moderada se mostró como un factor protector para el desarrollo de obesidad $\left(X^{2}=13^{\prime} 07\right.$; $\mathrm{p}=<0$ '001; Test exacto de Fisher; 0'0002; OR = 0'19; IC95\% de 0 ’07 a 0’48). La dieta hipercalórica fue un factor de riesgo para el desarrollo de obesidad $\left(\mathrm{X}^{2}=25^{\prime} 49 ; \mathrm{p}=<0\right.$ '001; Test exacto de Fisher: 3'57-07; OR = 9'68; IC95\% de 3,80 a 24,65). Para la tropa, la depresión fue un factor de riesgo para el desarrollo de obesidad $\left(\mathrm{X}^{2}=3,95 ; \mathrm{p}=<0,05\right.$; Test exacto de Fisher: 0'03; OR = 2' 52; IC95\% de 1 '00 a 6’33). La ansiedad y la asistencia al comedor no fueron factores de riesgo para el desarrollo de obesidad $\left(\mathrm{X}^{2}=\right.$ 0'04; $\mathrm{p}=0$ '83; Test exacto de Fisher: 0'5; OR= 0'91; IC95\% de 0'40 a 2'06; y X $\mathrm{X}^{2}=0$ '04; $\mathrm{p}=>0$ '05; Test exacto de Fisher: 0'05; $\mathrm{OR}=1$ '08; IC95\% de 0'48 a 2'45 respectivamente). (Tabla 3).

Se encontró que los menús del comedor de la Brigada proporcionaron en promedio $3.441 \mathrm{kcal}$. $(\mathrm{DE}=251, \mathrm{EE}=62$ '8).

\section{DISCUSIÓN}

La prevalencia de obesidad encontrada en este estudio (26'5 por cada 100 personas) resultó ser similar a la de la Encuesta Nacional de Salud y Nutrición 2006 (ENSANUT 2006) (24’2 por cada 100 personas), sin embargo los resultados no son comparables, ya que ésta define a la obesidad como IMC $\geq 30 \mathrm{~kg} / \mathrm{m}^{2}$.
Tabla 3. Análisis de los factores de riesgo para desarrollar obesidad en la $2^{a}$ Brigada de infanteria, marzo- abril 2010.

\begin{tabular}{|lcccccc|}
\hline Factor de riesgo & Caso & Control & $\mathbf{X}^{2}$ & $\mathbf{P}$ & Fisher & OR \\
\hline $\begin{array}{l}\text { Actividad fisica } \\
\text { moderada }\end{array}$ & 25 & 42 & 13.07 & 0.0003 & 0.0002 & 0.1900 \\
$\quad$ Tropa & 19 & 38 & 11.79 & 0.0005 & 0.0006 & 0.1900 \\
$\quad \begin{array}{l}\text { Oficiales } \\
\text { Jefes }\end{array}$ & 6 & 4 & 1.20 & 0.2733 & 0.4242 & 0 \\
Dieta & 0 & 0 & - & - & - & - \\
hipercalorica & 34 & 9 & 25.4998 & 0.0000 & 0.0000 & 9.6806 \\
$\quad \begin{array}{l}\text { Tropa } \\
\text { Oficiales }\end{array}$ & 26 & 8 & 20.2865 & 0.0000 & 0.0000 & 8.8214 \\
$\quad$ Jefes & 2 & 1 & 2.7429 & 0.0976 & 0.1515 & 9.0000 \\
Depresión & 34 & 27 & 2.0597 & 0.1512 & 0.1092 & 1.8102 \\
$\quad \begin{array}{l}\text { Tropa } \\
\text { Oficiales }\end{array}$ & 30 & 25 & 3.9584 & 0.0466 & 0.0381 & 2.5200 \\
$\quad$ Jefes & 1 & 2 & 0.1714 & 0.6788 & 0.5757 & 0.6000 \\
Ansiedad & 18 & 19 & 0.0429 & 0.8359 & 0.5000 & 0.9178 \\
$\quad \begin{array}{l}\text { Tropa } \\
\text { Oficiales }\end{array}$ & 17 & 18 & 0.1007 & 0.7510 & 0.4609 & 1.1498 \\
Jefes & 1 & 1 & 0.3000 & 0.5838 & 0.5757 & 0.4286 \\
$\begin{array}{l}\text { Asistencia al } \\
\text { comedor }\end{array}$ & 2 & 0 & - & - & - & - \\
$\begin{array}{l}\text { Tropa } \\
\text { Oficiales }\end{array}$ & 32 & 31 & 0.0429 & 0.8359 & 0.5000 & 1.0896 \\
Jefes & 25 & 29 & 0.0027 & 0.9585 & 0.5675 & 0.9770 \\
& 1 & 0 & - & - & - & - \\
\hline
\end{tabular}

La mayor edad encontrada en los casos podría explicar, en parte, que los mismos no realicen actividad física moderada en comparación con los controles, esto aunado a que las necesidades calóricas en ambos grupos son iguales, pero los casos consumen una dieta hipercalórica. Los militares que realizaron actividad física moderada tuvieron cinco veces menos riesgo de desarrollar obesidad que los que no la realizaron, por lo cual la actividad física moderada fue un factor protector. Resultados similares se encontraron en estudios en el estado de Morelos $^{17}$, y en el Estado de México ${ }^{37}$. Este resultado confirma nuestra hipótesis y refuerza la idea de que la actividad física moderada realizada, al menos 30 minutos en 3 ocasiones por semana es necesaria para evitar el desarrollo de obesidad; por tanto, no debe eliminarse de nuestras actividades diarias como miembros del Ejército mexicano, antes bien debe hacerse un esfuerzo por tener suficiente gente preparada en esta ámbito que guíe al resto de la población hacia la realización de ejercicios aeróbicos dirigidos.

Los militares que ingirieron una dieta hipercalórica tuvieron diez veces más riesgo de desarrollar obesidad que los que no la ingirieron, por lo que la dieta hipercalórica fue un factor de riesgo para el desarrollo de obesidad. Resultados similares se encontraron en el Distrito Federal ${ }^{12,38}$. Este resultado confirma nuestra hipótesis y refuerza la idea de que la dieta hipercalórica es un factor determinante para el desarrollo de obesidad $y$, por tanto, debe ponerse especial cuidado en el servicio de alimentación y las tiendas del interior de la institución, con la 
finalidad de no contribuir al desarrollo de obesidad en nuestra población.

Los militares de tropa, que presentaron depresión, tuvieron dos veces más riesgo de desarrollar obesidad que los que no la presentaron, por lo que la depresión, en la tropa, fue un factor de riesgo para el desarrollo de obesidad. Resultados similares se encontraron en un hospital del IMSS del Distrito Federal $^{38}$, Guadalajara ${ }^{39}$ y en Coahuila ${ }^{40}$. Este resultado confirma nuestra hipótesis y refuerza la idea de que, para la tropa, la depresión puede desencadenar obesidad, y por tanto debe ponerse especial cuidado en ésta y tratar de encontrar su causa para eliminarla.

La presencia de ansiedad no se asoció con el desarrollo de obesidad. Este resultado rechaza nuestra hipótesis y refuerza la idea de que, la ansiedad no desencadena obesidad en la población militar. Resultados similares se encontraron en un hospital del IMSS del Distrito Federal ${ }^{38}$.

La asistencia al comedor no se asoció con el desarrollo de obesidad. Este resultado rechaza nuestra hipótesis y sugiere que la asistencia al comedor no es un factor de riesgo para el desarrollo de obesidad en la población militar. No es posible comparar estos resultados, debido a que no se han hecho estudios similares en nuestra población.

Al comparar las calorías de los menús del servicio de alimentación (3.441 kcal.), encontramos que excedieron a las necesidades energéticas de esta población (2.536 kcal.), en 905 kilocalorías. Resultados similares se encontraron en la Escuela Militar de Enfermeras (menús de 3.750 kcal.)22 y en la Primera Brigada de Ingenieros Militares (necesidades de la población= $3.000 \mathrm{kcal}$., menús $=3.601$ '12 kcal. $)^{21}$. Este resultado confirma nuestra hipótesis y refuerza la idea de que los menús de la Segunda Brigada de Infantería se encuentran mal calculados, por tanto debe ponerse especial cuidado en ello, ya que podrían contribuir al aumento de la prevalencia de obesidad en el Ejército mexicano.

Como puntos débiles del estudio podría mencionar que no nos apegamos a la Norma Oficial Mexicana para el «tratamiento integral del sobrepeso y la obesidad», vigente a partir del 4 de Agosto del año 2010 (NOM-008-SSA3-2010), que define a la obesidad como IMC $\geq 30 \mathrm{~kg} / \mathrm{m}^{2}$ y al sobrepeso como IMC $>27 \mathrm{~kg} / \mathrm{m}^{2}$. ${ }^{41} \mathrm{Sin}$ embargo, por un lado esta norma se publicó posterior a la realización del estudio, y por otro lado no estamos de acuerdo con su utilización, ya que existen estudios en los que se ha demostrado que en nuestra población mexicana con un IMC de $27 \mathrm{~kg} / \mathrm{m}^{2}$ ya tiene mayor prevalencia de hiperinsulinemia, hiperglicemia, hipertrigiceridemia, diabetes e hipertensión arterial, además presentan un porcentaje de grasa similar al de obesidad que pacientes con IMC $\geq 30 \mathrm{~kg} / \mathrm{m}^{2}$ de otros países ${ }^{8}$. También como punto débil mencionaremos la imposibilidad de extrapolación de los resultados a la totalidad del Ejército mexicano, ya que este tipo de población militar (arma de infantería) está considerada como una de las que realizan mayor actividad física. Por último, otro punto débil es en relación a que comparamos con la población general las OR para los factores de riesgo, sin embargo no existen estudios de este tipo en población militar mexicana, por lo que es imperativo continuar con estudios como éste en la totalidad de la población militar a fin de poder contar con información más objetiva.

\section{CONCLUSIONES}

Aunque los resultados encontrados en este estudio podrían parecer predecibles ya que coinciden con otros artículos publicados, la importancia de los mismos radica en que en nuestro conocimiento, es el primer estudio con éstas características que se realiza en población militar mexicana, es por ello que sienta las bases para no seguir dependiendo de la información obtenida en otras poblaciones, lo cual nos permitirá hacer intervenciones más objetivas.

Por otro lado, sabemos que en ocasiones no existe la posibilidad de intervenir en todos los factores de riesgo, por diversos motivos como el económico, gracias a los resultados de este estudio podemos priorizar intervenciones en la población, atacando en primer lugar la ingesta hipercalórica, en segundo lugar promoviendo la actividad física, y en tercer lugar el origen de la depresión de la tropa.

Por último este estudio rompe el paradigma de que toda la población militar mexicana realiza actividad física moderada, justificando con esto el menú hipercalórico al que son expuestos. Sin embargo, gracias a este estudio, se demuestra que, aun tomando en cuenta su actividad física, los menús exceden en 905 kcal sus necesidades energéticas.

Estos resultados muestran un panorama alentador y gran campo de acción para la salud pública, ya que el Ejército Mexicano está a tiempo de revertir esta problemática si se llevan a cabo acciones adecuadas, puesto que la mayoría de los afectados no presentan un IMC de $30 \mathrm{~kg} / \mathrm{m}^{2}$.

\section{BIBLIOGRAFÍA}

1. Barquera-F S, Barquera S, García E, González-Villalpando C, Hernández-A M, Lonngi G, et al. Práctica Médica Efectiva: Obesidad en el adulto (E66). Instituto Nacional de salud Pública. México, 2003; 5 (2).

2. Obesidad y sobrepeso. Nota descriptiva No. 311. Organización Mundial de la Salud. 2006. Disponible en: http://www.who.int/mediacentre/factsheets/ fs311/es/print.html (Consultado: 07/06/09)

3. Morín R. Farmacoterapia de la Obesidad. $1^{\text {a }}$ edición. Editorial. 3-Litograpo, Academia Mexicana para el Estudio de la Obesidad. México, 2005:17-24.

4. Browman B, Rusell R. Conocimientos actuales sobre nutrición. OPS. Publicación 592. $8^{\mathrm{a}}$ ed. Washington, 2003:723-745.

5. Barquera S, Tolentino L, Dommarco R. Sobrepeso y obesidad: epidemiología, evaluación y tratamiento. $1^{\mathrm{a}}$ ed. Instituto Nacional de Salud Pública. México, 2006:31-163.

6. Olaiz-Fernández G, Rivera-Dommarco J, Shamah-Levy T, Rojas R, Villalpando-Hernández S, Hernández-Avila M, et al. Encuesta nacional de salud y nutrición (ENSANUT 2006). Instituto Nacional de Salud Pública. México, 2006.

7. García-García E, De la Llata-Romero M, Kaufer-Horwitz M, Tusié-Luna MT, Calzada-León R, Vázquez-Velázquez V, et al. La obesidad y el síndrome metabólico como problema de salud pública. Archivos de cardiología de México. México, 2008:318-337.

8. Carrasco F, Reyes E, Rimler O, Rios F. Exactitud del índice de masa corporal en la predicción de la adiposidad medida por impedanciometría bioeléctrica. Archivos Latinoamericanos de Nutrición. Chile, 2004;54(3):280-286.

9. Córdova V. Acuerdo Nacional para la Salud Alimentaria: Estrategia contra el Sobrepeso y la Obesidad. $1^{a}$ ed. México, 2009:7-18.

10. Tapia C. El Manual de Salud Pública. $2^{\mathrm{a}}$ ed. Editores intersistemas. México, 2006:1-55.

11. Marcos-Daccarett N, Núñez-Rocha G, Salinas-Martínez A, Santos-Ayarzagoitia M, Decanini-Arcaute H. Obesidad como factor de riesgo para trastornos metabólicos en adolescentes mexicanos. Revista de Salud Pública. México, 2007;9(2):180-193. 


\section{Hábitos alimentarios, actividad física y binomio ansiedad/depresión como factores de riesgo ...}

12. Ramos N. Obesidad en la población escolar y la relación con el consumo de comida rápida. Index de enfermería. México, 2006;5(55):1132-1296.

13. Tapia A, Masson L. Detección de síntomas depresivos en pacientes con sobrepeso y obesidad. Revista Chilena de Nutrición. Chile, 2006;2(33):162-169.

14. Reas D. Cognitive-Behavioral assisment of body image disturbances. Journal of psychiatric practice 2004;10:314-322.

15. Tapia A, Masson L. Detección de síntomas depresivos en pacientes con sobrepeso y obesidad. Revista Chilena de Nutrición. Chile, 2006;2(33):162-169.

16. Wilma B. Nutrición y vida activa: del conocimiento a la acción. OPS. Publicación 612. Washington, 2006:155-173.

17. Caballero C, Hernández B, Moreno H, Hernández-Girón C, Campero L, Cruz A, et al. Obesidad, actividad e inactividad física en adolescentes de Morelos, México: un estudio longitudinal. Archivos Latinoamericanos de Nutrición. México, 2007;57(3):231-237.

18. Robles S. Prevención clínica, guía para médicos. Pub. Cient. 568, OPS, 1998:130-141.

19. NOM-174-SSA1-1998: Para el manejo integral de la obesidad. México, 1998.

20. NOM-043-SSA2-2005: Servicios básicos de salud. Promoción y educación para la salud en materia alimentaria. Criterios para brindar orientación. México, 2005.

21. Ley del Instituto de Seguridad Social para las Fuerzas Armadas. Artículo 226. México, 2008:60-74. Disponible en: http://www.diputados.gob.mx/LeyesBiblio/pdf/84.pdf (Consultado: 14/07/09)

22. Chávez B. Estado nutricional de las alumnas de la Escuela Militar de Enfermeras; EMGS. México, 1996. Texto completo.

23. López M. Prevalencia de la obesidad y sus factores de riesgo en el personal masculino de oficiales y tropa en la $1^{\text {a }}$ Brigada de ingenieros del Campo Militar 1-A. EMGS. México, 1998. Texto completo.

24. Lizárraga T. Asociación del índice de masa corporal con alteraciones metabólicas en personal militar. Universidad del Ejército y Fuerza Aérea Mexicanos. Escuela Médico Militar. México, 2007. Texto completo.

25. Reyes J. Prevalencia del trastorno del espectro ansiedad y depresión en pacientes con obesidad. Universidad del Ejército y Fuerza Aérea Mexicanos. Escuela Médico Militar. México, 2007. Texto completo.

26. Archivo de la Dirección General de Sanidad Militar. Sección de planes y programas. Fuente directa. No publicado.

27. Pineda E. Metodología de la Investigación. OPS. $3^{\text {a }}$ ed. Washington, 2008:119-141.
28. Carrera R. Nociones sobre diseño de investigaciones médicas. Revista de Sanidad Militar. México, 1961;10:62-76.

29. Moreno L. Epidemiología clínica. $2^{\mathrm{a}}$ ed. Edit. Interamericana Mc.Graw-Hill. México, 1994:23-274.

30. Molinero L. Cuestionarios de Salud. Asociación de la Sociedad Española de Hipertensión Liga Española para la lucha contra la Hipertensión Arterial. España, 1998:1-5.

31. Arribas M. Diseño y validación de cuestionarios. Matronas profesión. España, 2004;5(17):23-29.

32. López-Alvarenga J, Vazquez-Velazquez V, Arcila-Martinez D, Sierra-Ovando A, Gonzalez-Baranco J, Sain-Pascual R. Exactitud y utilidad diagnóstica del Hospital Anxiety and Depression Scale (HAD) en una muestra de sujetos obesos mexicanos. Revista de investigación clínica. México, 2002;54(5):403-409.

33. Castillo-Salgado C. Módulos de principios de epidemiología para el control de enfermedades. $2^{\mathrm{a}}$ edición. OPS. Washington, 2002.3:52-62.

34. Castillo-Salgado C. Módulos de principios de epidemiología para el control de enfermedades; $2^{\mathrm{a}}$ edición; OPS. Washington, 2002. 5:35-42.

35. Silva L. Muestreo para la investigación en ciencias de la salud. Edit. Diaz de Santos. Cuba, 1993:39-60.

36. Mataix J. Necesidades energéticas del organismo. FUNIBER. España, 2008. II:57-80.

37. Cerecero P, Hernandez B, Aguirre D, Valdes R, Huitron G. Estilos de vida asociados al riesgo cardiovascular global en trabajadores universitarios del Estado de México. Salud Pública de México. México, 2009; 51(6):465-473.

38. Montes de Oca E, Loria J, Chavarria R. Prevalencia y factores de riesgo para el desarrollo de síndrome metabólico en personal médico de un servicio de urgencias de México. Revista cubana de medicina interna y emergencias. Mexico, 2008;7(3):1260-1272.

39. Colunga-Rodriguez C, Garcia de Alba J, Salazar-Estrada J, Angel-Gonzalez M. Diabetes tipo 2 y depresión en Guadalajara. Revista de Salud Pública. México 2008;10(1):137-149

40. Martínez-Mendoza J, Martinez-Ordaz V, Esquivel-Molina C, Velasco-Rodriguez V. Prevalencia de depresión y factores de riesgo en el adulto mayor hospitalizado. Revista médica del Instituto Mexicano del Seguro Social. México, 2007;45(1):21-28.

41. NOM-008-SSA3-2010, para el tratamiento integral del sobrepeso y la obesidad, México, 2010 